\title{
EFICÁCIA DOS FUNGICIDAS NO CONTROLE DE DOENÇAS DE FINAL DE CICLO (DFC) DA SOJA
}

\author{
Effectiveness of Fungicides for the Diseases Control in the \\ Soybean Final Cycle
}

Anderson Roberto Niero

Formando do Curso de Agronomia - PUCPR, trabalho de conclusão de curso. São José dos Pinhais - PR. E-mail:

Renato Tratch

Eng. Agrônomo, Msc. Professor do Curso de Agronomia - PUCPR, Curitiba - PR. E-mail: renato.tratch.pucpr.br.

Airton Rodrigues Pinto Junior

Professor Dr. do Curso de Agronomia da PUCPR, São José dos Pinhais - PR. E-mail: airton.junior.pucpr.br.

\begin{abstract}
Resumo
O experimento foi conduzido na Fazenda Experimental Gralha Azul - PUCPR, município de Fazenda Rio Grande, PR. O objetivo deste trabalho foi avaliar a eficiência de diferentes ingredientes ativos no controle de doenças de final de ciclo (DFC) da soja. A semeadura da cultivar COD 205 foi realizada no dia 15/11/02 em sistema de plantio direto. As sementes foram tratadas com fungicidas e inoculadas. A adubação foi de $300 \mathrm{~kg} \cdot \mathrm{ha}^{-1}$ de 00-20-30. O delineamento estatístico em blocos ao acaso foi utilizado, com oito tratamentos e quatro repetições. As médias foram comparadas pelo teste de Tukey a 5\%. A área útil para cada parcela foi de $6,72 \mathrm{~m}^{2}$. Os tratamentos foram: carbendazin $\left(0,250 \mathrm{~g}\right.$ i.a.ha $\left.{ }^{-1}\right)$, trifloxistrobina + propiconazol $\left(93,75+93,75 \mathrm{~g}\right.$ i.a.ha $\left.{ }^{-1}\right)$, tebuconazole $\left(0,150 \mathrm{~g}\right.$ i.a.ha $\left.\mathrm{h}^{-1}\right)$, pyraclostrobin $\left(75 \mathrm{~g}\right.$ i.a.ha $\left.\mathrm{h}^{-1}\right)$, fluquinconazol $\left(62,5 \mathrm{~g}\right.$ i.a.ha $\left.{ }^{-1}\right)$, epoxyconazole + pyraclostrobin $\left(137,25+183\right.$ g i.a.ha $\left.{ }^{-1}\right)$, fluquinconazole + carbendazim $(0,250$ $+62,5 \mathrm{~g}$ i.a.ha ${ }^{-1}$ ) e testemunha. Utilizou-se pulverizador costal propelido a $\mathrm{CO}_{2}, \mathrm{com}_{\text {barra de }} 4$ bicos tipo cônico cheio, espaçados de 50 cm, 2,6 bares de pressão e volume de calda de 288 L.ha ${ }^{-1}$. Aplicou-se no estádio fenológico R4 e avaliou-se no R7.1 por meio de escala diagramática, sendo considerada zero a ausência e cinco para situações acima de 70\% de área foliar lesionada. Os fungicidas flunquinconazole + carbendazin, pyraclostrobin, fluquinconazole e a epoxiconazole + pyraclostrobin diferiram em relação aos demais tratamentos. A produtividade e a massa de mil sementes não diferiram estatisticamente. O fluquinconazole apresentou 100\% de controle de oídio.
\end{abstract}

Palavras-chave: Glycine max; Septoria glycines; Cercospora kikuchi. 


\begin{abstract}
The experiment was conducted in the Gralha Azul Experimental Farm - PUCPR, in Fazenda Rio Grande - PR. The objective of this experiment was to evaluate the efficiency of different active ingredients in the disease control of end of cycle of the soy. The seeding, of cultivar COD 205, was realized in the day 15/11/02 under no-tillage system. The seeds was treated with fungicides and inoculated. The fertilization was $300 \mathrm{~kg} \cdot \mathrm{ha}^{-1}$ 00-20-30. Randomized block experiment was used to compare the treatments composed by eight treatments and four replications. The data was submitted to the analysis of variance and to the Tukey test $5 \%$ to compare the means. The useful area of each plot was $6.72 \mathrm{~m}^{2}$. The treatments were: carbendazin $\left(0.250 \mathrm{~g}\right.$ i.a.h $\left.\mathrm{a}^{-1}\right)$, trifloxistrobina + propiconazol $\left(93.75+93.75\right.$ g i.a.ha $\left.a^{-1}\right)$, tebuconazole $\left(0.150 \mathrm{~g}\right.$ i.a. $\left.\mathrm{ha}^{-1}\right)$, pyraclostrobin $(75 \mathrm{~g}$ i.a.h $\left.a^{-1}\right)$, fluquinconazole $\left(62.5 \mathrm{~g}\right.$ i.a.h $\left.\mathrm{a}^{-1}\right)$, epoxyconazole + pyraclostrobin $\left(137.25+183\right.$ g i.a.ha $\left.a^{-1}\right)$, fluquinconazole + carbendazim $\left(0.250+62.5 \mathrm{~g}\right.$ i.a.h $\left.\mathrm{a}^{-1}\right)$ and a control. A costal spray propelled by $\mathrm{CO}_{2}$, with bars of four full cone nozzles, spaced $50 \mathrm{~cm}$, pressure of 2.6 bars and spraying suspension volume of $288 \mathrm{~L}$.ha ${ }^{-1}$. It was applied in phenologic state $R 4$ and evaluated in $R 7.1$ by diagrammatic scales, being zero absence and five above $70 \%$ of diseased leaf area. The fungicides fluquinconazole + carbendazin, pyraclostrobin, fluquinconazole and epoxiconazole + pyraclostrobin presented different results comparing to the other treatments. There was no statistical difference in productivity and in the weight of 1000 seeds. The fluquinconazole presented $100 \%$ of Oidium control.
\end{abstract}

Keywords: Glycine max; Septoria glycines; Cercospora kikuchi.

\title{
INTRODUÇÃO
}

A soja (Glycine max (L.) Merr.) teve rápida expansão no Brasil nos últimos 30 anos, devido ao seu valor econômico e graças ao desenvolvimento, por meio de melhoramento genético e de novas cultivares mais adaptadas às condições do país. A safra mundial em 1999/2000 foi estimada em 155 milhões de toneladas de grão, sendo o Brasil o segundo maior produtor. No entanto, essa expansão da cultura aliada à falta de cuidado fitossanitário permitiu que a maioria dos patógenos se disseminasse, por meio da semente, pelas regiões produtoras (KLINGELFUSS; YORINORI, 2001a).

Cerca de 40 doenças já foram registradas na cultura, no Brasil, as quais são responsáveis por prejuízos anuais que chegam a 15-20\% da produção total do país. Algumas podem causar 100\% de perdas "podridão parda" (Phialophora gregata) (HENNING et al., 1997). As doenças de final de ciclo (DFC) podem causar perdas superiores a 20\% (KLINGELFUSS; YORINORI, 2001b).

No ano de 1994, os prejuízos devido a doenças, nos dez países maiores produtores de soja, foram estimados em três bilhões de dólares. Fungos como Septoria glycine (mancha parda), Cercospora kikuchii (crestamento foliar de Cercospora) e outras espécies de fungos causaram perdas de quase 700 milhões de dólares nos dez países maiores produtores de soja, das quais $70 \%$ foram observadas no Brasil. No Brasil, na safra de 1997/98, as perdas foram estimadas em 1,3 bilhões de dólares (KLINGELFUSS; YORINORI, 2001b).

A mancha parda e o crestamento foliar de Cercospora são conhecidos como doenças de final de ciclo (DFC) por ocorrerem com maior severidade na fase final de granação da soja. Entre as principais medidas de controle dessas doenças estão a utilização de sementes sadias, o tratamento de sementes, a incorporação de restos de culturais, a aplicação de fungicidas entre o florescimento e enchimento de grãos e a rotação de cultura com espécies não suscetíveis (KLINGELFUSS; YORINORI, 2001a).

A mancha parda é a doença mais amplamente disseminada no país, podendo causar severa redução no rendimento. Os sintomas são provenientes de infecção na semente, aparecem cerca de duas semanas após a emergência, como pequenas manchas de contorno angulares, castanho-avermelhada, nas folhas unifolioladas. Sob situações favoráveis, a doença pode atingir os primeiros trifolíolos e causar severa desfolha em plantas de até 35-40 dias. Após esse período, as plantas se recuperam. Um novo surto da 
doença reaparece no final da fase de enchimento de vagens, após o estádio R6. Nas folhas verdes, surgem pontuações pardas, menores de $1 \mathrm{~mm}$ de diâmetro, que evoluem e formam manchas com halos amarelados, de coloração parda na página superior e rosada na página inferior, medindo de 1 a $3 \mathrm{~mm}$ de diâmetro (HENNING et al., 1997).

O crestamento foliar de cercospora (Cercorspora kikuchii) ocorre em regiões de clima é quente e chuvoso. O fungo ataca todas as partes da planta e pode ser responsável por severas reduções do rendimento e da qualidade da semente. Nas folhas, os sintomas aparecem a partir do final de granação e são caracterizados por pontuações escuras e castanho-avermelhadas, as quais coalescem e formam grandes manchas escuras que resultam em severo crestamento foliar ou desfolha prematura. Nas vagens, aparecem pontuações vermelhas que evoluem para manchas castonho-avermelhadas. Pela vagem, o fungo atinge a semente e causa a mancha púrpura no tegumento. Nas hastes, o fungo causa manchas vermelhas, geralmente superficiais, limitada ao córtex (HENNING et al., 1997).

O oídio (Microsphaeria difusa) iniciou sua expressão a partir da safra 1995/96 e era considerada de pouca expressão, sendo observada, principalmente, em sojas tardias, na região Sul. Esse fungo é um parasita obrigatório que se desenvolve em toda a parte aérea da soja, como folhas, hastes, pecíolos e vagens, porém é mais visível nas folhas. O sintoma é expresso pela presença do fungo nas partes atacadas e caracterizado por uma fina camada de micélio e conídios pulverulentos que, de pequenos pontos brancos, podem cobrir toda parte aérea da planta, com menos severidade nas vagens. Nas folhas, ao longo do tempo, a coloração branca do fungo muda para castanho-acinzentado, dando a aparência de sujeira nas duas fases da folha. Sob condições de infecção severa, a cobertura de micélios e a frutificação do fungo, além do dano direto ao tecido, impedem a fotossíntese e as folhas secam e caem prematuramente, dando aspecto de soja dessecada por herbicida. A infecção pode ocorrer em qualquer estágio de desenvolvimento da planta, mas é mais visível na fase de floração e quanto mais cedo iniciar a infecção, maior será o efeito da doença sobre o rendimento (EMBRAPA, 1998).

Embora a resistência genética seja a forma mais econômica e eficaz do controle de doenças, não há cultivar resistente para a maioria delas e o controle químico está sendo uma alternativa para o controle de doenças como mancha parda e crestamento foliar de Cercospora (KLINGELFUSS; YORINORI, 2000c).

Prade et al. (2002a,b,c), Souza Neto e Zagonel (2002), Forcelini et al. (2002), Utimata (2002), Godoy et al. (2002) e Juliatti et al. (2002) descrevem sobre a eficiência e a necessidade de se controlar as DFC, tendo em vista as perdas ocasionadas por essas doenças.

O objetivo deste trabalho foi avaliar a eficiência de diferentes ingredientes ativos no controle de doenças de final de ciclo (DFC) da soja.

\section{MATERIAL E MÉTODOS}

O experimento foi conduzido na Fazenda Experimental Gralha Azul, pertencente à PUCPR,

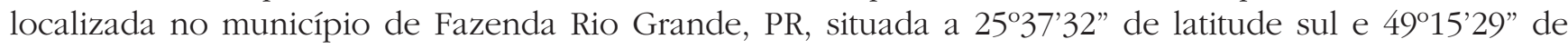
longitude oeste e altitude de 920 m. A região, segundo a classificação de Koëpen, apresenta clima do tipo Cfb e solo pertencente à unidade de mapeamento associação Latossolo Vermelho-Amarelo Álico + Cambissolo Álico, com horizonte A proeminente textura argilosa, fase subtropical, relevo suave ondulado.

O experimento foi conduzido com a cultivar COD 205, que apresenta características de suscetibilidade as DFC (Septoria glycines e Cercospora kikuchii). A semeadura foi realizada no dia 15 de novembro em semeadura direta com histórico de área com rotação de soja-milho nos anos anteriores. As sementes foram tratadas com thiabendazole + thiram ( $17 \mathrm{~g}+70 \mathrm{~g}$ i.a. para $100 \mathrm{~kg}$ de sementes) e inoculadas com inoculante turfoso (500 g para $50 \mathrm{~kg}$ de sementes). A adubação foi feita com $300 \mathrm{~kg} \cdot \mathrm{ha}^{-1}$ do formulado 00-20-30, segundo a análise de solo e histórico de área. Os herbicidas aplicados foram tepraloxydin $\left(0,51 \cdot \mathrm{ha}^{-1}\right)$, lactofen $\left(0,2401 \cdot \mathrm{ha}^{-1}\right)$ e chloransulam-metil (40g.ha- $\left.{ }^{-1}\right)$ e o inseticida aplicado foi metamidophos $\left(0,51 \cdot \mathrm{ha}^{-1}\right)$. 
As parcelas constaram de 10 linhas de 5 metros cada, espaçadas de $0,4 \mathrm{~m}$ e com 16 sementes por metro linear. A área útil para cada parcela foi de $6,72 \mathrm{~m}^{2}$, constituída por seis linhas centrais e desprezando $1,10 \mathrm{~m}$ de cabeceira como bordadura.

O delineamento estatístico utilizado foi em blocos ao acaso, constando de oito tratamentos com quatro repetições. Os dados foram submetidos à análise com teste de comparação de médias de Tukey a 5\%. Os tratamentos aplicados foram: carbendazin $\left(0,250\right.$ g i.a.ha $\left.{ }^{-1}\right)$, trifloxistrobina + propiconazol $\left(93,75+93,75\right.$ g i.a.ha $\left.{ }^{-1}\right)$, tebuconazole $\left(0,150\right.$ g i.a.ha $\left.{ }^{-1}\right)$, pyraclostrobin $\left(75\right.$ g i.a.ha $\left.{ }^{-1}\right)$, fluquinconazol $\left(62,5\right.$ g i.a.ha $\left.{ }^{-1}\right)$, epoxyconazole + pyraclostrobin $\left(137,25+183\right.$ g i.a.ha $\left.a^{-1}\right)$, fluquinconazole + carbendazim $\left(0,250+62,5\right.$ g i.a.ha $\left.{ }^{-1}\right)$ e testemunha, com pulverizador costal, propelido a $\mathrm{CO}_{2}$, equipado com uma barra com 4 bicos do tipo cônico cheio, espaçados com $50 \mathrm{~cm}$ sob uma pressão de 2,6 bares, que proporciona um volume de calda de 288 L.ha ${ }^{-1}$. As aplicações ocorreram no estádio fenológico R4. Para verificar a eficiência dos tratamentos foram feitas avaliações da severidade da doença por meio de escala descritiva no estágio R7.1 (início a 50\% das folhas amareladas).

A severidade de doenças foliares de final de ciclo no campo foi determinada por meio de escala de notas de 0 a 5, sendo 0- ausência de área foliar (AF) infectada, 1- de 1 a 10\% de AF infectada, 2- 11 a 30\% de AF infectada, 3- 31 a 50\% de AF infectada, 4- 51 a 70\% de AF infectada e 5- mais de 70\% de AF infectada. A severidade de oídio (Microsphaeria difusa) foi determinada via área foliar lesionada e convertida em área sob a curva de progresso da doença.

Foi determinada a produtividade e a massa de 1000 sementes em cada um dos tratamentos, sendo corrigidos a 13\% de umidade.

\section{RESULTADOS E DISCUSSÃO}

Os fungicidas testados apresentaram diferença estatística significativa em relação à testemunha no que se refere à área foliar lesionada (TABELA 1). Os fungicidas flunquinconazole + carbendazin $\left(0,250+62,5 \mathrm{~g}\right.$ i.a.ha $\left.\mathrm{a}^{-1}\right)$, pyraclostrobin $\left(75 \mathrm{~g}\right.$ i.a.ha $\left.\mathrm{h}^{-1}\right)$, fluquinconazole $\left(62,5 \mathrm{~g}\right.$ i.a.ha $\left.{ }^{-1}\right)$ e a epoxiconazole + pyraclostrobin $\left(137,25\right.$ + 183 g i.a.ha $\left.{ }^{-1}\right)$ diferiram em relação aos demais, representando a menor severidade da doença. Dados similares foram obtidos por Venancio et al. (2002), quando avaliaram dose de carbendazim, tebuconazole, pyraclostrobin e pyraclostrobin + epoxiconazole. Yorinori et al. (2000) obtiveram resultados similares testando tebuconazole e carbendazin.

A produtividade não diferiu estatisticamente dos tratamentos com fungicidas e a testemunha (TABELA 1). Yorinori et al. (2000) relatam que alguns trabalhos com uso de fungicidas apresentam redução na severidade, porém não ocorreram diferenças significativas na produtividade. Os prováveis fatores que interferiram na severidade das doenças de final de ciclo foram os baixos índices pluviométricos (FIGURA 1) e as temperaturas baixas (FIGURA 2) após a aplicação dos fungicidas. Sinclair e Backman (1993) citam que para a Cercospora kikuchii e Septoria glycines a temperatura entre $28-30^{\circ} \mathrm{C}$ e longos períodos de umidade favorecem o desenvolvimento da doença.

A massa de mil sementes não diferiu estatisticamente entre si (TABELA 1). Yorinori et al. (2000), usando a cultivar BR -37 , verificaram maior valor de massa de mil sementes no tratamento com tebuconazole.

Para o controle de oídio, o ingrediente ativo que apresentou 100\% de controle da doença foi o fluquinconazole (62,5 g i.a.ha ${ }^{-1}$ ). Venancio et al. (2002) verificaram que o tratamento com pyraclostrobin (100 g i.a.ha-1), usando a cultivar BR - 16, apresentou melhor controle que os demais produtos. 
TABELA 1 - Efeito de fungicidas nas doenças de final de ciclo (DFC), produtividade e peso de mil sementes na cultura da soja, safra 2002/03, Fazenda Rio Grande - PR

Table 1 - Fungicides effect in the final cycle diseases, productivity and weight of a thousand seeds in the soybean culture, harvest 2002/03, Fazenda Rio Grande-PR

\begin{tabular}{|c|c|c|c|c|c|c|}
\hline & \multicolumn{2}{|c|}{ DFC $^{1}$} & \multicolumn{2}{|l|}{ Oidio } & \multirow{2}{*}{$\begin{array}{l}\text { Produtividade }^{2} \\
\text { kg.ha' }\end{array}$} & $\begin{array}{l}\text { massa } \\
1000 \\
\text { sementes }^{2} \\
\text { gr }\end{array}$ \\
\hline Carbendazim & 2,75 & $\mathrm{~b}$ & 171,5 & $\mathrm{~b}$ & & 146,0517 \\
\hline Trifloxistrobina + Propiconazol & 2,5 & $\mathrm{bc}$ & 37,63 & $\mathrm{~cd}$ & 2878 & 137,0517 \\
\hline Tebuconazole & 2,5 & $\mathrm{bc}$ & 13,13 & $\mathrm{~cd}$ & 2781 & 169,977 \\
\hline Pyraclostrobin & 1,5 & $\mathrm{~cd}$ & 47,25 & c & 2738 & 160,3218 \\
\hline Fluquinconazole & 1,75 & bcd & 0,00 & $\mathrm{~d}$ & 2721 & 131,1379 \\
\hline Epoxiconazole + Pyraclostrobin & 1,25 & $\mathrm{~d}$ & 19,25 & $\mathrm{~cd}$ & 2569 & 159,0402 \\
\hline Fluquinconazole + Carbendazim & 1,75 & bcd & 8,875 & $\mathrm{~cd}$ & 2824 & 148,6667 \\
\hline TESTEMUNHA & 4,5 & $\mathrm{a}$ & 973,0 & a & 2771 & 142,2759 \\
\hline CV (\%) & \multicolumn{2}{|c|}{20,84} & \multicolumn{2}{|l|}{$\mathbf{1 1 , 0 7}$} & 14,32 & 11,32 \\
\hline $\mathbf{F}$ & \multicolumn{2}{|c|}{18,38} & \multicolumn{2}{|c|}{1440,29} & & $0,27^{\text {ns }} \quad 2,53^{\text {ns }}$ \\
\hline
\end{tabular}

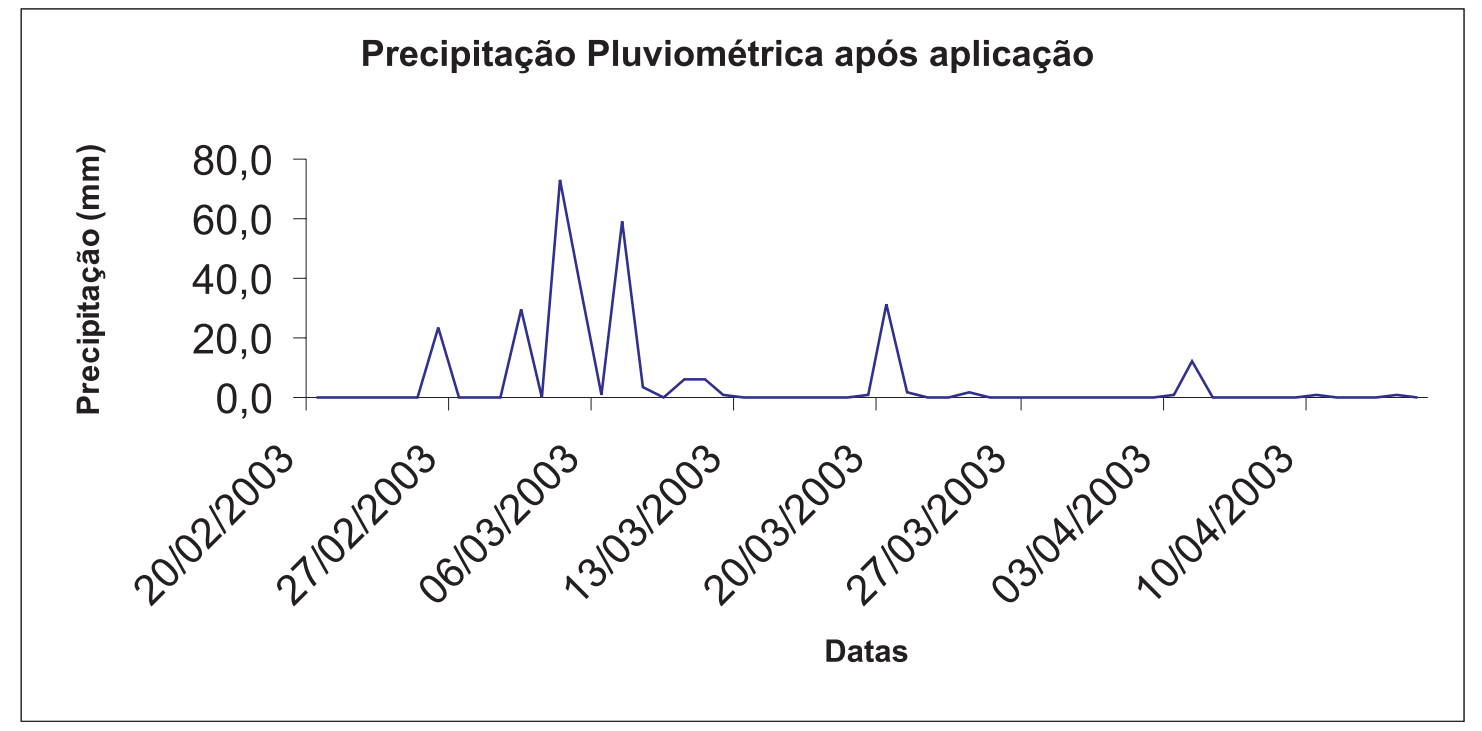

GRÁFICO 1 - Precipitação entre 20/02/03 e 10/04/03, Fazenda Experimental Gralha Azul, Fazenda Rio Grande, PR

Graph 1 - Precipitation between 20/02/03 and 10/04/03, Gralha Azul Experimental Farm, Fazenda Rio Grande, PR 


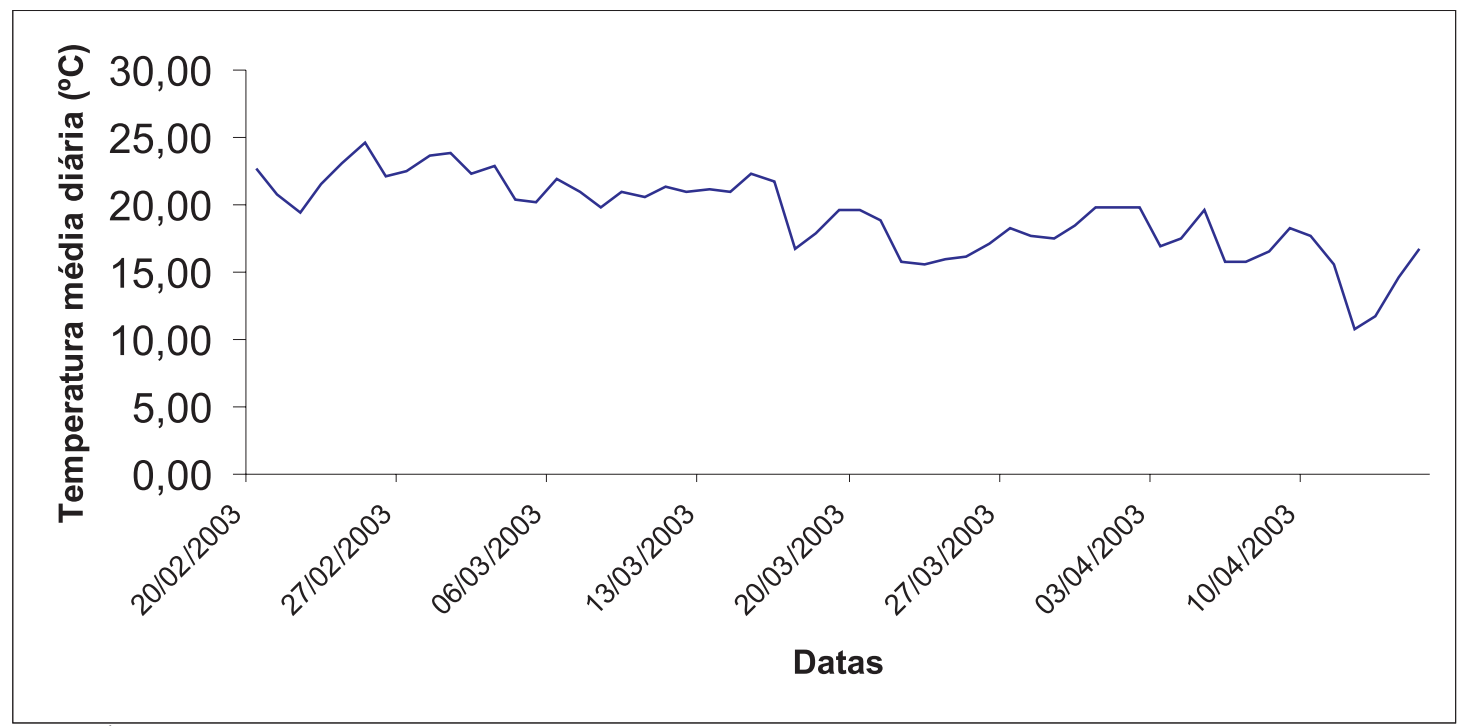

GRÁFICO 2 - Temperaturas médias diárias de 20/02/03 a 10/04/03, Fazenda Experimental Gralha Azul, Fazenda Rio Grande, PR

Graph 2 - Daily average temperatures bewtween 20/02/03 and 10/04/03, Gralha Azul Experimental Farm, Fazenda Rio Grande, PR

\section{CONCLUSÕES}

A aplicação dos fungicidas flunquinconazole + carbendazin $\left(0,250+62,5\right.$ g i.a.ha $\left.{ }^{-1}\right)$, pyraclostrobin $\left(75\right.$ g i.a.ha $\left.{ }^{-1}\right)$, fluquinconazole $\left(62,5\right.$ g i.a.ha $\left.{ }^{-1}\right)$ e a epoxiconazole + pyraclostrobin $\left(137,25+183\right.$ g i.a.ha a $\left.^{-1}\right)$ resultou em menor severidade da doença de final de ciclo.

O fungicida fluquinconazole $\left(62,5 \mathrm{~g}\right.$ i.a.ha- $\left.{ }^{-1}\right)$ promoveu a ausência de sintomas para o oídio.

Os fungicidas trifloxistrobina + propiconazol $\left(93,75+93,75\right.$ g i.a.ha $\left.a^{-1}\right)$, tebuconazole $(0,150 \mathrm{~g}$ i.a.ha $\left.\mathrm{a}^{-1}\right)$, epoxyconazole + pyraclostrobin $\left(137,25+183 \mathrm{~g}\right.$ i.a.ha $\left.{ }^{-1}\right)$, fluquinconazole + carbendazim $(0,250+$ 62,5 g i.a.ha- ${ }^{-1}$ ) promoveram redução na severidade da doença.

A produtividade e a massa de mil sementes não foram afetadas pela ocorrência das doenças de final de ciclo.

\section{AGRADECIMENTO}

Agradeço a minha família pela motivação em relação a esse desafio e também ao mestre Prof. Renato Tratch, que sempre esteve disposto a auxiliar. Agradeço também aos colegas Karol Geovani Czelusniak, Nilmar Coelho Zeni, ao Eng. Agr. Maurício Cesar Iung e ao funcionário Rogério Cotarski, que auxiliaram na condução do experimento.

\section{REFERÊNCIAS}

EMBRAPA. Centro Nacional de Pesquisa de Soja. Recomendações técnicas para a cultura da soja no Paraná 1998/99. Londrina: EMBRAPA-Soja, 1998. 201 p. (EMBRAPA - CNPSo. Documentos, 119).

FORCELINI, C. A. et al. Controle de doenças foliares em soja em função de fungicidas e sua época de aplicação. In: CONGRESSO BRASILEIRO DE SOJA, 2, 2002, Foz do Iguaçu. Resumos... Londrina: EMBRAPASoja, 2002. p. 43. 
GODOY, C. V. et al. Avaliação da eficiência de fungicidas no controle das doenças de final de ciclo causadas por Septoria glycines e Cercospora kikuchii, e do oídio na cultura da soja. In: CONGRESSO BRASILEIRO DE SOJA, 2, 2002, Foz do Iguaçu. Resumos... Londrina, EMBRAPA-Soja, 2002. p. 49.

HENNING, A. A.; CAMPO, R. J.; SFREDO, G. J. Tratamento com fungicidas, aplicação de micronutrientes e inoculação de sementes de soja. Londrina: EMBRAPA-CNPSO, 1997. 6 p. (Comunicado Técnico, 58).

JULIATTI, F. C.; FONTES, R. C.; HAMAWAKI, T. Eficiência de fungicidas sistêmicos no controle de doenças de final de ciclo na cultura da soja. In: CONGRESSO BRASILEIRO DE SOJA, 2., 2002, Foz do Iguaçu.

Resumos... Londrina: EMBRAPA-Soja, 2002. p. 52.

KLINGELFUSS, L. H.; YORINORI, J. T. Infecção latente de Colletotrichum truncatum e Cercospora Kikuchii e efeito de fungicidas sobre doenças de final de ciclo da soja. Summa Phytophatologica, v. 26, n. 1, p. 356-361, 2001a.

Infecção latente de Colletotrichum truncatum e Cercospora Kikuchii em soja. Fitopatologia Brasileira, v. 26, n. 1, p. 158-164, 2001b.

PRADE, A. G.; TAVELA, V.; BALARDIN, R. S. Aplicações de fungicidas no controle de doenças de final de ciclo em soja. In: CONGRESSO BRASILEIRO DE SOJA, 2., 2002, Foz do Iguaçu. Resumos... Londrina: EMBRAPA-Soja, 2002a. p. 32.

Controle de doenças de final de ciclo em soja no Paraná. In: CONGRESSO BRASILEIRO DE SOJA, 2., 2002, Foz do Iguaçu. Resumos... Londrina: EMBRAPA-Soja, 2002b. p. 33.

Controle químico de doenças em soja no Paraná. In: CONGRESSO BRASILEIRO DE SOJA, 2, 2002, Foz do Iguaçu. Resumos... Londrina: EMBRAPA-Soja, 2002c. p. 38.

SINCLAIR, J. B.; BACKMAN, P. A. Compendium of soybean diseases. St. Paul: APS Press, 1993. p. 106.

SOUZA NETO, A. M.; ZAGONEL, J. Eficiência dos fungicidas azoxystrobin e carbendazin aplicados em diferentes épocas no controle de doenças de final de ciclo na cultura da soja. In: CONGRESSO BRASILEIRO DE SOJA, 2, 2002, Foz do Iguaçu. Resumos... Londrina: EMBRAPA-Soja, 2002. p. 41.

UTIAMADA, C. M.; SATO, L. N.; TORRES, J. P. Eficiência de fungicidas no controle de mancha parda e crestamento foliar de Cercospora na cultura da soja. In: CONGRESSO BRASILEIRO DE SOJA, 2., 2002, FOz do Iguaçu. Resumos... Londrina: EMBRAPA-Soja, 2002. p. 48.

VENANCIO, W. S. et al. Avaliação da eficiência de diferentes grupos de fungicidas no controle de oidio na cultura da soja. In: CONGRESSO BRASILEIRO DE SOJA, 2., 2002, Foz do Iguaçu. Resumos... Londrina: EMBRAPA-Soja, 2002. p. 91.

YORINORI, J. T. et al. Levantamento das doenças fúngicas da soja, seus impactos sobre rendimento e aferição das atuais medidas de controle. In: EMBRAPA. Resultados de pesquisas da Embrapa soja 1999. Londrina: EMBRAPA-Soja, 2000. p. 64-71. 
